\title{
The Tell 'Uqair temple (4th mill.BC): colours and iconography
}

This contribution focuses on the "painted temple" of Tell "Uqair (Babylonia) during the late Uruk period (3400-3100 cal. BC). This building that has provided evidences for bright paintings on the interior. Based on the archaeological report, as well as on in-depth comparisons with other urukean building, we propose a colorful reconstruction of the paintings, together with new interpretations of its iconography and symbolic meaning. 